\title{
Bedeutung der wahrgenommenen Motivierungsqualität für intrinsische Motivation und Selbstkonzept von Grundschulkindern
}

\author{
Chantal Lepper $(\mathbb{D}) \cdot$ Justine Stang $(\mathbb{D}) \cdot$ Nele McElvany $(\mathbb{D}$
}

Eingegangen: 25. November 2020 / Überarbeitet: 12. Mai 2021 / Angenommen: 26. Mai 2021 / Online publiziert: 1. Juli 2021

(C) Der/die Autor(en) 2021

Zusammenfassung Ein qualitätsvoller Unterricht ist von zentraler Bedeutung sowohl für leistungsbezogene als auch für motivationale Lernergebnisse von Schülerinnen und Schülern. Die Motivierungsqualität des Unterrichts als eine bedeutsame Facette der Basisdimension konstruktive Unterstützung wurde bisher eher selten untersucht. Um die Bedeutung der wahrgenommenen Motivierungsqualität für motivationale Merkmale von Grundschulkindern zu erforschen, wurde basierend auf Angaben von Viertklässlerinnen und -klässlern $(N=187)$ analysiert, inwiefern die Motivierungsqualität des Mathematikunterrichts neben der Mathematiknote prädiktiv für die intrinsische Motivation und das Fähigkeitsselbstkonzept der Lernenden ist. Auch wurde geprüft, ob dem Kompetenzerleben der Lernenden eine vermittelnde Funktion zukommt. Die Ergebnisse zeigten, dass die wahrgenommene Motivierungsqualität neben der Mathematiknote ein bedeutsamer Prädiktor für beide motivationalen Merkmale war. Während das Kompetenzerleben die Zusammenhänge zwischen der Motivierungsqualität bzw. der Mathematiknote und dem Fähigkeitsselbstkonzept bedeutsam vermittelte, wurden die Zusammenhänge für die intrinsische Motivation der Schülerinnen und Schüler wiederum nicht mediiert. Resümierend werden die Befunde hinsichtlich möglicher Implikationen für zukünftige Forschung und Praxis diskutiert.

\footnotetext{
Chantal Lepper $(\bowtie) \cdot$ Justine Stang $\cdot$ Nele McElvany

Institut für Schulentwicklungsforschung, Technische Universität Dortmund, Vogelpothsweg 78, 44227 Dortmund, Deutschland

E-Mail: chantal.lepper@tu-dortmund.de

Justine Stang

E-Mail: justine.stang@tu-dortmund.de

Nele McElvany

E-Mail: office.mcelvany-ifs.fk12@tu-dortmund.de
} 
Schlüsselwörter Grundschule · Intrinsische Motivation · Motivierungsqualität • Selbstkonzept · Unterrichtsqualität

\title{
Importance of perceived motivational support for intrinsic motivation and self-concept of elementary students
}

\begin{abstract}
High instructional quality is of central importance for students' motivational and performance-related learning outcomes. The motivational quality of instruction as a significant facet of the basic dimension constructive support has rarely been studied. To investigate the relevance of perceived motivational quality for elementary students' motivational indicators, based on data from fourth-grade students $(N=187)$ it was analyzed whether motivational support in mathematics lessons is predictive for students' intrinsic motivation and academic self-concept in math when math grade was taken into account. It was also examined whether students' feeling of competence was a significant mediator of these relations. The results showed that perceived motivational support was a significant predictor of both motivational variables beyond math grade. While students' feeling of competence significantly mediated the relations between motivational support respectively math grade and self-concept, the relations with intrinsic motivation were not mediated. Concluding, findings are discussed in terms of future implications for research and practice.
\end{abstract}

Keywords Elementary school · Instructional quality · Intrinsic motivation · Motivational support $\cdot$ Self-concept

\section{Einleitung}

Die Qualität von Unterricht stellt eine wichtige Rahmenbedingung für erfolgreiche Lernprozesse von Schülerinnen und Schülern dar (Einsiedler 2017). Eine höher ausgeprägte Unterrichtsqualität ist dabei nicht nur mit besseren Leistungen, sondern auch mit günstigeren Ausprägungen motivationaler Merkmale von Schülerinnen und Schülern in verschiedenen Domänen und Unterrichtsfächern verknüpft (Kunter und Trautwein 2013; Praetorius et al. 2018). Sowohl in der Grundschule als auch im weiterführenden Schulkontext wurde bei der Erforschung des Zusammenspiels zwischen Unterrichtsqualität und multikriterialen Lernergebnissen vornehmlich auf die drei Basisdimensionen Klassenführung, kognitive Aktivierung und konstruktive Unterstützung fokussiert (Grundschule: Decristan et al. 2016; Fauth et al. 2014; Stang und McElvany 2020; weiterführende Schule: Baumert et al. 2010; Klieme und Rakoczy 2008; Lipowsky et al. 2009). Wie Grundschulkinder die Motivierungsqualität des Unterrichts, die als eine spezifische Facette der konstruktiven Unterstützung gilt, wahrnehmen, wurde bislang seltener untersucht. Motivationale Merkmale von Schülerinnen und Schülern nehmen einen hohen Stellenwert für das Lernen im schulischen Kontext ein, da sie nicht nur für die aktuelle Lernbereitschaft, sondern auch für bildungsrelevante Entscheidungen und Bildungsverläufe bedeutsam sind (z. B. Lauermann et al. 2017). Daher stellt die Unterstützung zentraler motivationaler Merkma- 
le, wie der intrinsischen Motivation und des Fähigkeitsselbstkonzepts, ein wichtiges Ziel von Unterricht dar (Kunter 2005). Angesichts des allgemein zu verzeichnenen Absinkens motivationaler Merkmale im Verlauf der Schulzeit (Musu-Gillette et al. 2015; Scherrer und Preckel 2019) sollte der Motivierungsqualität des Unterrichts und ihrer Bedeutung für motivationale Merkmale auch in der Grundschule verstärkt Aufmerksamkeit zukommen. Während die Relevanz von Leistungsrückmeldungen, die häufig mithilfe von Schulnoten erfolgen, für verschiedene Bedürfnisfacetten des Selbstbestimmungserlebens und motivationale Merkmale bereits mehrfach gezeigt werden konnte (Arens 2019; Rakoczy 2008; Viljaranta et al. 2014; Weidinger et al. 2017, 2019), wurde das Zusammenspiel zwischen wahrgenommener Motivierungsqualität des Unterrichts und motivationalen Merkmalen bei Grundschulkindern bislang nur vereinzelt untersucht (Stang et al. 2020). Die vorliegende Studie verfolgt daher das Ziel, die Motivierungsqualität des Mathematikunterrichts aus der Sicht von Grundschulkindern zu erforschen und zu prüfen, inwiefern bedeutsame Zusammenhänge zwischen der Motivierungsqualität des Unterrichts und verschiedenen motivationalen Merkmalen bestehen. Außerdem wird die Mathematiknote als eine typische Form der Leistungsrückmeldung in der Schule als weitere Determinante motivationaler Merkmale berücksichtigt. Darüber hinaus wird untersucht, ob die Zusammenhänge über das Kompetenzerleben der Lernenden im Unterricht bedeutsam vermittelt werden.

\section{Theoretischer Hintergrund}

\subsection{Motivierungsqualität als Facette konstruktiver Unterstützung}

Die Motivierungsqualität des Unterrichts gilt als wichtiger Indikator von Unterrichtsqualität und kann als spezifisches Merkmal der konstruktiven Unterstützung im Unterricht definiert werden (Helmke 2012; Klieme et al. 2006). Unter dem Begriff Unterrichtsqualität werden Merkmale subsummiert, die sich auf die Prozessebene bzw. die Tiefenstruktur des Unterrichts beziehen und bedeutsam mit leistungsbezogenen und motivationalen Lernergebnissen verknüpft sind (Decristan et al. 2020; Kunter und Voss 2011; Seidel und Shavelson 2007). Im deutschsprachigen Raum hat sich eine dreigliedrige Konzeptualisierung der Unterrichtsqualität etabliert, in der die drei generischen Basisdimensionen Klassenführung, kognitive Aktivierung und konstruktive Unterstützung differenziert werden (Fauth et al. 2014; Klieme et al. 2009; Kunter et al. 2013; Praetorius et al. 2020). Ähnliche theoretische Rahmenmodelle existieren auch im internationalen Forschungskontext (z. B. Pianta und Hamre 2009). Die Klassenführung beschreibt, inwiefern es Lehrkräften im Unterricht gelingt, Unterrichtsstörungen präventiv zu vermeiden, um die Lernzeit bestmöglich für Lernprozesse zu nutzen (Hochweber et al. 2014; Kuger 2016). Die kognitive Aktivierung fokussiert auf die Anregung vertiefter Denkprozesse von Schülerinnen und Schülern im Unterricht (Baumert et al. 2010; Lipowsky et al. 2009).

Die konstruktive Unterstützung, die für motivationale Merkmale von Schülerinnen und Schülern besonders relevant ist, umfasst Gestaltungsmerkmale, wie ein wertschätzendes Lernklima, fürsorgliche Beziehungen zwischen der Lehrkraft und 
den Lernenden, gegenseitigen Respekt und einen konstruktiven Umgang mit Fehlern im Unterricht (Kunter et al. 2013; Patrick et al. 2011; Reeve und Jang 2006). Dabei werden sowohl affektiv-motivationale als auch strukturierende Aspekte des Unterrichts als relevant erachtet, wie positive Beziehungen zwischen Lernenden und Lehrkraft oder Hilfestellungen bei der Bearbeitung von kognitiv herausfordernden Aufgaben (Patrick et al. 2007; Ruzek et al. 2016; Wentzel et al. 2010). Die empirische Befundlage verdeutlicht, dass die wahrgenommene konstruktive Unterstützung von Lernenden unterschiedlicher Altersgruppen prädiktiv für motivationale Merkmale von Lernenden in verschiedenen Domänen war (Dietrich et al. 2015; Fauth et al. 2014; Roorda et al. 2011). Auch in Bezug auf den Mathematikunterricht ergaben Studien, dass Lernende, die sich im Unterricht stärker konstruktiv unterstützt fühlten, eine höhere intrinsische Motivation für das Fach Mathematik oder ein günstigeres Fähigkeitsselbstkonzept in Mathematik aufwiesen (Lazarides und Buchholz 2019; Lazarides und Ittel 2012; Sakiz et al. 2012; Scherer et al. 2017; Schiepe-Tiska et al. 2016). Festzuhalten ist allerdings, dass es an Befunden für den Primarschulkontext eher mangelt.

Die Motivierungsqualität des Unterrichts gilt als eine spezifische Facette der konstruktiven Unterstützung (Klieme et al. 2006). Sie spiegelt wider, inwiefern es im Unterricht gelingt, die Lernmotivation von Schülerinnen und Schülern anzuregen und aufrecht zu erhalten (Kunter und Trautwein 2013). Dabei spielt das Selbstbestimmungserleben der Lernenden beim Lernen eine wichtige Rolle, das beispielsweise durch Freude an einer Aktivität oder Interesse an Inhalten angetrieben werden kann (Ryan und Deci 2020). Darüber hinaus zielt die Motivierungsqualität des Unterrichts auf die Bestärkung des Vertrauens in die eigenen Fähigkeiten ab (Kunter et al. 2013).

Die Motivierungsqualität stellt ein facettenreiches Konstrukt dar, sodass motivationale Merkmale von Lernenden auf vielfältige Weise im Unterricht gefördert werden können (Kunter 2005; Ohle und McElvany 2016). Eine spezifische Facette der Motivierungsqualität ist die Interessensanregung auf Seiten der Lernenden (Assor et al. 2002; Krapp 2002). Das Interesse von Schülerinnen und Schülern gilt als eine wichtige Quelle intrinsisch motivierter Lernprozesse. So können beispielsweise der Einbezug persönlicher Interessen der Lernenden, die Kontextualisierung von Lerninhalten durch Lebensweltbezug und die Verdeutlichung von Relevanz dazu beitragen, motivationale Merkmale wie Interesse und intrinsische Motivation zu steigern (Gaspard et al. 2015; Høgheim und Reber 2015; Laufs und Kempert 2021; Rakoczy et al. 2008). Darüber hinaus stellt eine geeignete Passung zwischen den Aufgabenanforderungen und den Fähigkeiten der Lernenden eine weitere Facette der Motivierungsqualität dar. Das erfolgreiche Bewältigen von Aufgaben kann zum Beispiel zu einem höheren Kompetenzerleben im Unterricht beitragen (Rakoczy 2008; Ryan und Deci 2020). Weitere Facetten der Motivierungsqualität sind ein enthusiastisches, empathisches und verständnisvolles Lehrkraftverhalten, die Schaffung von Wahlmöglichkeiten im Unterricht sowie sachlich-konstruktive Rückmeldungen der Lehrkraft (Aelterman et al. 2019; Jang et al. 2010; Keller et al. 2014; Rakoczy 2008). Insgesamt lässt sich festhalten, dass die Motivierungsqualität des Unterrichts viele verschiedene Aspekte einer motivierenden Unterrichtsgestaltung umfasst und über die motivationale Unterstützung durch die Lehrperson hinausgeht. Aufgrund der 
Vielzahl an Merkmalen von Motivierungsqualität werden in Studien häufig nur einzelne Aspekte betrachtet. In der vorliegenden Arbeit wird insbesondere auf die Anregung von Interesse und die Vermittlung von Freude am Unterricht fokussiert, um motivationale Schülerinnen- und Schülermerkmale zu adressieren (Pintrich 2003).

\subsection{Motivationale Merkmale von Lernenden}

Die Relevanz motivationaler Merkmale liegt nicht nur in der bedeutsamen Verknüpfung mit akademischen Leistungen, sondern auch in einer positiven Erlebensqualität der Lernenden begründet, beispielsweise der erlebten Freude beim Lernen (Arens et al. 2017; Marsh et al. 2016; Ryan und Deci 2020). Die intrinsische Motivation und das Fähigkeitsselbstkonzept gelten als zwei zentrale motivationale Merkmale, die wichtig für gelingende Lernprozesse sind.

Die intrinsische Motivation bezeichnet eine bestimmte Qualität der Motivation, bei der Lernprozesse oder Aktivtäten beispielsweise aufgrund von empfundener Freude oder Interesse an Lerninhalten selbstbestimmt angetrieben werden (Ryan und Deci 2020). Für intrinsisch motivierte Lernende sind äußere Beweggründe, wie die soziale Anerkennung der Eltern, eher nachrangig. Intrinsisch motivierte Aktivitäten werden demnach an sich als belohnend und positiv wahrgenommen. So lassen sich intrinsische und extrinsische Motivationsformen voneinander unterscheiden. Allerdings lassen sich auch bei der extrinsischen Motivation verschiedene Formen finden, die stärker selbstbestimmt sind (Ryan und Deci 2020). Bei der identifizierten und integrierten Regulation fungieren stärker internale Anreize, wie die persönliche Bedeutsamkeit, die Wertschätzung oder Nützlichkeit, als bedeutsame Antreiber der extrinsischen Motivation. Im Gegensatz zur intrinsischen Motivation müssen die Handlungen bei der identifizierten und der integrierten Motivationsform jedoch nicht zwingend als positiv erlebt werden. So kann beispielsweise die Vorbereitung auf eine Prüfung, die persönlich bedeutsam ist, zwar selbstbestimmt sein, jedoch wenig Freude bereiten.

Verschiedene Forschungsbefunde verdeutlichen, dass sich stärker intrinsisch motivierte Schülerinnen und Schüler nicht nur häufiger mit Lerninhalten beschäftigen, sondern Lernaktivitäten auch häufiger aus eigener Motivation fortsetzen, sodass die Kompetenzentwicklung sowie die Interessensbildung begünstigt werden (Eccles und Wigfield 2002; Krapp und Hascher 2014; Taylor et al. 2014). Für Grundschulkinder wurde gezeigt, dass die intrinsische Motivation der Lernenden für das Fach Mathematik positiv mit der Mathematikleistung zusammenhing (Viljaranta et al. 2014; Weidinger et al. 2017). Dabei ergaben Studien, dass die zuvor rückgemeldete Leistung bedeutsam für die intrinsische Motivation der Grundschulkinder in Mathematik war (Arens 2019; Garon-Carrier et al. 2016). Zudem konnten bedeutsame Zusammenhänge zwischen dem Kompetenzerleben der Lernenden im Unterricht und der intrinsischen Motivation gefunden werden (Corpus et al. 2009; Taylor et al. 2014).

Das Fähigkeitsselbstkonzept umfasst selbstbezogene kognitive und affektive Fähigkeitsüberzeugungen der Lernenden in einer bestimmten Domäne (Eccles und Wigfield 2002; Marsh 1990). Für die Genese und Aufrechterhaltung des Fähigkeitsselbstkonzepts fungieren leistungsbezogene Kriterien als zentrale Informationsquellen, beispielsweise Schulnoten. Der Zusammenhang zwischen rückgemeldeten Leis- 
tungen und Fähigkeitsselbstkonzept kann anhand sozialer und dimensionaler Vergleiche der Lernenden beschrieben werden (Internal/External Frame of Reference Model; Marsh 1990). Anhand von Studienbefunden für Lernende der Sekundarstufe lässt sich festhalten, dass das Fähigkeitsselbstkonzept bedeutsam mit den schulischen Leistungen von Lernenden verknüpft ist (Möller et al. 2014; Niepel et al. 2014). Zudem liegen Befunde für ein wechselseitiges Verhältnis zwischen akademischen Leistungen und dem Fähigkeitsselbstkonzept vor (Arens et al. 2017; Marsh et al. 2016; Sewasew und Schroeders 2019). Für das Fach Mathematik ließen sich ebenfalls reziproke Befundmuster bei Grundschulkindern finden (Weidinger et al. 2018). Bedeutsame Zusammenhänge wurden auch in längsschnittlichen Studiendesigns gezeigt (Viljaranta et al. 2014). Außerdem berichteten Lernende mit einem günstigeren Fähigkeitsselbstkonzept von einem höheren Kompetenzerleben im Unterricht und einer höheren intrinsischen Motivation in der entsprechenden Domäne (Birkel und Tarnai 2018; Skaalvik und Skaalvik 2013; Taylor et al. 2014; Weidinger et al. 2018).

\subsection{Zusammenhang von Motivierungsqualität mit motivationalen Merkmalen und Bedeutung des Kompetenzerlebens}

Während in verschiedenen Studien für die Basisdimension konstruktive Unterstützung gezeigt wurde, dass positive Zusammenhänge zwischen der konstruktiven Unterstützung und motivationalen Merkmalen, wie der intrinsischen Motivation oder dem Fähigkeitsselbstkonzept, vorlagen (z.B. Dietrich et al. 2015; Lazarides und Buchholz 2019; Lazarides und Ittel 2012), existieren bislang nur vereinzelt Befunde zur Relevanz der Motivierungsqualität des Unterrichts für motivationale Merkmale von Grundschulkindern. In TIMSS 2019 wurde die Motivierungsqualität des Mathematikunterrichts im Grundschulkontext erforscht (Stang et al. 2020). Dabei zeigte sich ein positiver Zusammenhang zwischen der individuell wahrgenommenen Motivierungsqualität des Mathematikunterrichts und der intrinsischen Motivation der Lernenden vierter Klassen. In einer weiteren Studie wurde die positive Verknüpfung zwischen der wahrgenommenen Motivierungsqualität und der intrinsischen Motivation im Fach Mathematik auch für Lernende weiterführender Schulen gezeigt (Thomas und Andreitz 2021). Kleickmann, Steffensky und Praetorius (2020) fokussierten in einer Studie im naturwissenschaftsbezogenen Sachunterricht auf die Aspekte Lehrerfürsorglichkeit, positives Feedback und Autonomieunterstützung als motivationsförderliche Bedingungen im Unterricht und fanden einen positiven $\mathrm{Zu}$ sammenhang zum domänenspezifischen Interesse von Viertklässlerinnen und Viertklässlern. Allerdings basieren die Ergebnisse auf einzelnen Unterrichtseinheiten.

In einem heuristischen Rahmenmodell von Klieme und Rakoczy (2008) wird für das Zusammenspiel zwischen der konstruktiven Unterstützung und motivationalen Merkmalen angenommen, dass die Effekte über verschiedene Komponenten des Selbstbestimmungserlebens der Lernenden im Unterricht vermittelt werden. Vor diesem Hintergrund und in Anlehnung an die Selbstbestimmungstheorie (Ryan und Deci 2020) kann ebenfalls abgeleitet werden, dass die Zusammenhänge zwischen der Motivierungsqualität und motivationalen Merkmalen über das Kompetenzerleben der Schülerinnen und Schüler im Unterricht vermittelt werden. Der 
Selbstbestimmungstheorie zufolge stellt das Kompetenzerleben von Lernenden eine wichtige motivationspsychologische Bedürfniskomponente dar. Das Kompetenzerleben beschreibt, inwiefern sich Schülerinnen und Schüler in der Interaktion mit ihrer Lernumwelt als fähig und wirksam wahrnehmen. Dabei können verschiedene Aspekte der Motivierungsqualität für das Kompetenzerleben der Lernenden relevant sein. So kann die Auswahl von Aufgaben mit optimalem Anforderungsniveau dazu beitragen, dass eine gelungene Passung zwischen den Fähigkeiten der Lernenden und den zu bearbeitenden Aufgaben vorliegt, sodass die Lernenden ein gesteigertes Kompetenzerleben erfahren (Aelterman et al. 2019; Reeve und Jang 2006). Darüber hinaus können transparente Leistungserwartungen und sachlich-konstruktive Leistungsrückmeldungen förderlich für das Kompetenzerleben sein (Niemiec und Ryan 2009; Rakoczy et al. 2013; Wang und Eccles 2013). Interessant gestaltete Unterrichtsinhalte können ebenfalls wichtige Voraussetzungen für das Kompetenzerleben der Lernenden sein (Benning et al. 2019). Durch das Anknüpfen an persönliche Interessen und die kindliche Lebenswelt der Schülerinnen und Schüler wird an bestehendes Vorwissen angeknüpft, welches das Kompetenzerleben im Unterricht begünstigen kann (Pintrich 2003; Schraw et al. 2001; Schiepe-Tiska et al. 2016).

Die empirische Befundlage verdeutlicht, dass die Basisdimension konstruktive Unterstützung eine günstige Bedingung für die Erfüllung motivationaler Bedürfnisfacetten des Selbstbestimmungserlebens darstellt (z. B. Jang et al. 2010; Sakiz et al. 2012; Sierens et al. 2009). In Bezug auf das Kompetenzerleben von Schülerinnen und Schüler weiterführender Schulen zeigten Rakoczy et al. (2008) positive Zusammenhänge zur wahrgenommenen Unterstützung im Mathematikunterricht. Außerdem ergaben weitere Studien für den Sekundarschulkontext, dass das Kompetenzerleben im Mathematikunterricht relevant für motivationale Merkmale von Lernenden war (Kunter 2005; Lazarides und Raufelder 2017; Schukajlow und Krug 2014). Das Kompetenzerleben im Unterricht wurde hinsichtlich einer möglichen Vermittlungsfunktion für die Zusammenhänge zwischen der Motivierungsqualität des Unterrichts und motivationalen Merkmalen von Grundschulkindern allerdings kaum untersucht.

Hingegen ist das Zusammenspiel von Leistungsrückmeldungen, wie Schulnoten, Kompetenzerleben und motivationalen Merkmalen besser erforscht. Im ErwartungsWert-Modell (Wigfield und Eccles 2002) werden Schulnoten als wichtige kompetenzbezogene Erfahrung von Lernenden betrachtet, die bedeutsam mit motivationalen Merkmalen verbunden sind. Neben den bereits angeführten Studienresultaten zu signifikanten Zusammenhängen zwischen Schulnoten und zentralen motivationalen Merkmalen (z. B. Arens 2019; Garon-Carrier et al. 2016; Weidinger et al. 2018, 2019), existieren außerdem Befunde, die die Bedeutung von Leistungsrückmeldungen für das Kompetenzerleben der Lernenden verdeutlichen (Jang et al. 2012; Rakoczy et al. 2013). Für die Erforschung der Motivierungsqualität und ihrer Relevanz für das Kompetenzerleben und motivationale Merkmale ist es sinnvoll, die Schulnote als typische Form der Leistungsrückmeldung zusätzlich als weiteren Faktor zu berücksichtigen. 


\section{Fragestellungen}

Motivationale Merkmale von Lernenden gelten als wichtige Zielkriterien von Unterricht (Kunter 2005). In dem vorliegenden Beitrag wurde untersucht, inwiefern die individuell wahrgenommene Motivierungsqualität des Unterrichts bedeutsam mit zentralen motivationalen Schülerinnen- und Schülermerkmalen verbunden ist und inwiefern die Zusammenhänge über das Kompetenzerleben der Grundschulkinder im Unterricht vermittelt werden. Dabei wurde die Mathematiknote als ein wichtiger Prädiktor von motivationalen Merkmalen berücksichtigt. Folgende Forschungsfragen leiteten das Erkenntnisinteresse:

Forschungsfrage 1 (F1) Ist die individuell wahrgenommene Motivierungsqualität des Unterrichts unter gleichzeitiger Berücksichtigung der Mathematiknote prädiktiv für a) die intrinsische Motivation und b) das Fähigkeitsselbstkonzept der Grundschulkinder im Fach Mathematik?

Forschungsfrage 2 (F2) Werden die Zusammenhänge zwischen der individuell wahrgenommenen Motivierungsqualität des Unterrichts und a) der intrinsischen Motivation sowie b) dem Fähigkeitsselbstkonzept im Fach Mathematik unter Berücksichtigung der Mathematiknote durch das Kompetenzerleben im Unterricht mediiert?

Es wurde angenommen, dass die individuell wahrgenommene Motivierungsqualität unter Berücksichtigung der Mathematiknote sowohl bedeutsam mit der intrinsischen Motivation (Hypothese 1a) als auch mit dem Fähigkeitsselbstkonzept der Lernenden (Hypothese 1b) im Fach Mathematik verknüpft ist. In Anlehnung an das heuristische Rahmenmodell von Klieme und Rakoczy (2008) zum Zusammenspiel von Unterrichtsqualität und multikriterialen Lernergebnissen und an die Selbstbestimmungstheorie (Ryan und Deci 2020) wurde außerdem erwartet, dass das Kompetenzerleben der Lernenden die Bedeutung der individuell wahrgenommenen Motivierungsqualität bzw. der Mathematiknote für die intrinsische Motivation (Hypothese 2a) und für das Fähigkeitsselbstkonzept (Hypothese $2 b$ ) bedeutsam vermittelt.

\section{Methode}

\subsection{Stichprobe}

An der vorliegenden Studie nahmen 187 Viertklässlerinnen und Viertklässler aus 12 Grundschulklassen in Nordrhein-Westfalen teil ${ }^{1}$. Die Grundschulkinder waren im Mittel zehn Jahre alt $(M=10,39 ; S D=0,44)$ und in etwa die Hälfte der Lernenden war weiblich (48,4\%). Ungefähr ein Drittel aller Lernenden (30,3\%) gab an, zuhause neben Deutsch auch eine andere Sprache zu sprechen.

\footnotetext{
${ }^{1}$ Eine A-priori Analyse zur erforderlichen Stichprobengröße ergab, dass bei angestrebter mittlerer Effektstäke mindestens 137 Teilnehmende für die anvisierten Strukturgleichungsmodelle erforderlich waren.
} 


\subsection{Instrumente}

Die wahrgenommene Motivierungsqualität des Unterrichts wurde anhand von fünf Items erfasst, welche insbesondere auf die Interessensanregung und Vermittlung von Freude im Unterricht als wichtige Facetten der Motivierungsqualität (vgl. Abschnitt 2.1) fokussierten. Bei den Items handelt es sich um eine Modifikation der Items aus der BiSS-EvalLesen Studie (McElvany et al. 2017), welche wiederum auf Items aus der Pythagoras-Eingangsbefragung basierten (Rakoczy et al. 2005). Die modifizierte Skala zur Erfassung der Motivierungsqualität wies im Kontext anderer Studien gute Reliabilitätswerte auf (Igler et al. 2019; $\alpha=0,85$; Stang et al. in Druck; $\alpha=0,89$ ). Die wahrgenommene Motivierungsqualität wurde beispielsweise anhand der Items ,Im Mathematikunterricht kann unsere Lehrerin bzw. unser Lehrer uns Schülerinnen und Schüler manchmal richtig für die Inhalte begeistern." und „Im Mathematikunterricht gestaltet unsere Lehrerin/unser Lehrer die Inhalte sehr spannend." gemessen $(\alpha=0,61)$. Zur Beantwortung der Items wurde eine vierstufige Likert-Skala verwendet ( $1=$,trifft nicht zu“ bis $4=$,trifft zu“).

Die intrinsische Motivation und das Fähigkeitsselbstkonzept wurden mittels bereits erprobter Skalen erhoben (Trends in International Mathematics and Science Study 2015: Selter et al. 2016). Die intrinsische Motivation wurde anhand von neun Items ermittelt und wies eine gute interne Konsistenz auf $(\alpha=0,91)$. Beispielitems lauten „Ich mag Aufgaben mit Zahlen“ und „Ich freue mich auf den Mathematikunterricht.“. Das Fähigkeitsselbstkonzept, welches selbstbezogene Fähigkeitseinschätzungen in Mathematik umfasst, wurde mithilfe von sieben Items erfasst (z. B. „Normalerweise bin ich gut in Mathematik.“, „Ich kann schwierige Mathematikaufgaben gut lösen."). Die Reliabilität der Skala war ebenfalls gut $(\alpha=0,87)$. Die Items zur intrinsischen Motivation und zum Fähigkeitsselbstkonzept wurden jeweils anhand einer vierstufigen Likert-Skala beantwortet $(1=$, ,stimmt gar nicht“ bis $4=$,stimmt genau").

Das Kompetenzerleben der Lernenden wurde durch vier Items erfasst, welche explizit auf das Erleben von Kompetenz durch kompetenzunterstützende Unterrichtsbedingungen Bezug nehmen. Die Skala wurde basierend auf etablierten Skalen zum Selbstbestimmungserleben im Unterricht von Chen et al. (2015) und von Reeve und Sickenius (1994) entwickelt. Ein Beispielitem lautet „In unserem Mathematikunterricht habe ich den Eindruck, dass ich erfolgreich schwierige Aufgaben lösen kann“ $(\alpha=0,74){ }^{2}$ Lernende schätzten ihr Kompetenzerleben im Unterricht auf einer vierstufigen Likert-Skala ein ( $1=$,stimmt gar nicht“ bis $4=$,stimmt genau“).

Zudem wurden die Grundschulkinder gebeten, ihre zuletzt erhaltene Mathematiknote $\mathrm{zu}$ berichten $(1=$ sehr gut bis $6=$ ungenügend). In den Analysen wurden die Werte nicht rekodiert, sodass kleine Werte guten Noten entsprechen und beispiels-

\footnotetext{
2 Konfirmatorische Faktorenanalysen zeigten, dass die Konstrukte Fähigkeitsselbstkonzept in Mathematik und Kompetenzerleben im Unterricht zwei statistisch trennbare Konstrukte darstellen. Der Modellvergleich zwischen einem Ein-Faktor-Modell $\left(\chi^{2}=68,61, d f=42, \mathrm{CFI}=0,95\right.$, RMSEA =0,06) und einem Zwei-Faktoren-Modell $\left(\chi^{2}=59,59, d f=41, \mathrm{CFI}=0,97\right.$, RMSEA $\left.=0,05\right)$ ergab keine signifikante Modellverschlechterung $\left(\Delta_{\chi}^{2}=9,02, \Delta_{d f}=1, p \geq 0,05\right)$. Es wurde das Modell gewählt, das den theoretischen Annahmen der Trennbarkeit entsprach und besser zu den Daten passte.
} 
weise eine negative Korrelation zwischen der Mathematiknote und dem Fähigkeitsselbstkonzept erwartet wurde.

\subsection{Durchführung}

Die papierbasierte Befragung wurde von geschulten Testleiterinnen und Testleitern durchgeführt, wobei die Fragebögen weitestgehend selbstständig von den Lernenden bearbeitet wurden. Neben den Fragen zur wahrgenommenen Motivierungsqualität im Mathematikunterricht und zum Kompetenzerleben im Unterricht, machten die Lernenden Angaben zu den motivationalen Merkmalen in Bezug auf Mathematik und zu ihrer zuletzt erhaltenen Zeugnisnote im Fach Mathematik. Die Lernenden berichteten zudem demographische Angaben. Die Bearbeitung des Fragebogens dauerte ca. $15 \mathrm{~min}$. Die Studienteilnahme war freiwillig und erfolgte mit Einverständnis der Eltern.

\subsection{Analysestrategie}

Zur Beantwortung der Forschungsfragen wurden Strukturgleichungsmodelle in Mplus 8 (Muthén und Muthén 1998-2017) berechnet. Die Analysen fokussieren auf die individuelle Wahrnehmung der Motivierungsqualität aus Sicht der Lernenden, da eine Überprüfung der Intra-Klassen-Korrelationen (ICCs) geringe Werte ergab (ICCs $\leq 0,06)$. Um die geclusterte Struktur der Daten trotzdem zu berücksichtigen, wurde die Option type=complex gewählt und die Klassenzugehörigkeit als Clustervariable spezifiziert. Außerdem wurden alle eingesetzten Konstrukte am Klassenmittelwert zentriert, sodass individuelle Werte im Verhältnis zum Mittelwert der Mitschülerinnen und Mitschüler interpretiert werden. Die Konstrukte Motivierungsqualität, Kompetenzerleben, intrinsische Motivation und Fähigkeitsselbstkonzept wurden latent modelliert.

Zur Bewertung der Strukturgleichungsmodelle wurden der Chi-Quadrat-Wert $\left(\chi^{2}\right)$, die Freiheitsgrade $(d f)$, der Comparative Fit Index (CFI) und der Root Mean Square Error of Approximation (RMSEA) herangezogen. Dabei indizierten CFI-Werte gröBer als 0,95 und RMSEA-Werte kleiner als 0,06 (Hu und Bentler 1999) eine gute Modellpassung. Der Anteil fehlender Werte lag mit Ausnahme der Mathematiknote zwischen 1,0\% und 7,2\% (Anteil fehlender Werte Mathematiknote: 28,3\%). Eine Analyse möglicher Muster fehlender Werte nach Little (1988) ergab, dass die fehlenden Werte zufällig verteilt waren (MCAR-Test: $\chi^{2}=2512,06, d f=2605, p=0,90$ ). Fehlende Werte wurden mit Full Information Maximum Likelihood (FIML) geschätzt. Alle Analysen wurden unter Verwendung des robusten Standardfehlerschätzers (MLR) durchgeführt (Muthén und Satorra 1995).

In einem ersten Modell wurde geprüft, ob die Motivierungsqualität des Unterrichts und die Mathematiknote jeweils prädiktiv für die intrinsische Motivation und das Fähigkeitsselbstkonzept waren (F1). Es wurden direkte Pfade zur Vorhersage der abhängigen Variablen intrinsische Motivation und Fähigkeitsselbstkonzept durch die unabhängigen Variablen Motivierungsqualität und Mathematiknote modelliert. Dabei wurden Korrelationen zwischen den Prädiktorvariablen (Motivierungsqualität 
und Mathematiknote) sowie zwischen den abhängigen Variablen (intrinsische Motivation und Fähigkeitsselbstkonzept) zugelassen.

In einem zweiten Modell wurde das Kompetenzerleben als Mediator der $\mathrm{Zu}$ sammenhänge zwischen wahrgenommener Motivierungsqualität und intrinsischer Motivation bzw. Fähigkeitsselbstkonzept sowie der Zusammenhänge zwischen Mathematiknote und intrinsischer Motivation bzw. Fähigkeitsselbstkonzept spezifiziert (F2). Dabei wurden neben direkten Pfaden zur Vorhersage der abhängigen Variablen intrinsische Motivation und Fähigkeitsselbstkonzept durch die wahrgenommene Motivierungsqualität und die Mathematiknote vier indirekte Pfade zur Vermittlung der Zusammenhänge über das Kompetenzerleben in das Modell aufgenommen. Korrelationen zwischen den Prädiktoren sowie zwischen den Zielkriterien wurden jeweils zugelassen. Von einer vollständigen Mediation der Effekte wurde ausgegangen, wenn zuvor signifikante Effekte der direkten Pfade über die indirekten Pfade vermittelt wurden, sodass diese nach Berücksichtigung des Mediators nicht mehr signifikant waren. Eine partielle Mediation lag hingegen vor, wenn sich direkte und indirekte Pfade zugleich als statistisch signifikant erwiesen (MacKinnon et al. 2007). Es werden jeweils standardisierte Regressionskoeffizienten berichtet.

\section{Ergebnisse}

\subsection{Deskriptive Kennzahlen und bivariate Korrelationen}

Die Grundschulkinder schätzten die wahrgenommene Motivierungsqualität des Mathematikunterrichts, die intrinsische Motivation und das Fähigkeitsselbstkonzept in der mathematischen Domäne sowie das Kompetenzerleben im Mathematikunterricht als positiv ausgeprägt ein (siehe Tab. 1). Die Mittelwerte dieser Variablen lagen jeweils über dem theoretisch erwartbaren Mittel $(M=2,5$, siehe Tab. 2). Zudem zeigte sich eine eher geringe Varianz in den Einschätzungen der Lernenden hinsichtlich der wahrgenommenen Motivierungsqualität sowie den motivationalen Merkmalen. Die durchschnittliche Mathematiknote der Lernenden lag bei einem ,gut“. Die Motivierungsqualität stand in einem signifikant negativen Zusammenhang mit der Mathematiknote $(r=-0,27)$, sodass Lernende mit besseren Noten die Motivierungsqualität durchschnittlich höher einschätzten. Die Stärke des Zusammenhangs war moderat (Cohen 1992). Es zeigte sich zudem ein statistisch bedeutsamer und stark positiver

Tab. 1 Deskriptive Kennzahlen und Korrelationen der manifesten Skalen

\begin{tabular}{llllllll}
\hline & $M$ & $S D$ & 1 & 2 & 3 & 4 & 5 \\
\hline 1 Motivierungsqualität & 3,21 & 0,58 & 1 & $0,55^{*}$ & $0,35^{*}$ & $0,49^{*}$ & $-0,27^{*}$ \\
2 Intrinsische Motivation & 3,30 & 0,70 & - & 1 & $0,62^{*}$ & $0,52^{*}$ & $-0,40^{*}$ \\
3. Fähigkeitsselbstkonzept & 3,27 & 0,72 & - & - & 1 & $0,66^{*}$ & $-0,56^{*}$ \\
4. Kompetenzerleben & 3,16 & 0,63 & - & - & - & 1 & $-0,56^{*}$ \\
5. Mathematiknote $^{\mathrm{a}}$ & 2,04 & 0,91 & - & - & - & - & 1 \\
\hline
\end{tabular}

$* p<0,05$

aNotenstufen $1=$ sehr gut bis $6=$ ungenügend 
Tab. $2 t$-Test Ergebnisse

\begin{tabular}{llllll}
\hline & $M$ & $S D$ & $\mathrm{~T}$ & $d f$ & $p$ \\
\hline 1 Motivierungsqualität & 3,21 & 0,58 & 16,62 & 185 & $<0,01$ \\
2 Intrinsische Motivation & 3,30 & 0,70 & 13,83 & 185 & $<0,01$ \\
3. Fähigkeitsselbstkonzept & 3,27 & 0,72 & 14,08 & 172 & $<0,01$ \\
4. Kompetenzerleben & 3,16 & 0,63 & 14,24 & 184 & $<0,01$ \\
\hline
\end{tabular}

Testwert $M=2,5$, Vierstufige Antwortskala für alle aufgeführten Instrumente $(1=$,stimmt gar nicht“ bis $4=$,stimmt genau“)

Zusammenhang zwischen der intrinsischen Motivation und dem Fähigkeitsselbstkonzept in Mathematik $(r=0,62)$. Je höher die selbstberichtete intrinsische Motivation ausfiel, desto günstiger waren auch die Angaben zum Fähigkeitsselbstkonzept (siehe Tab. 1).

\subsection{Wahrgenommene Motivierungsqualität als Prädiktor für motivationale Merkmale}

Das erste Strukturgleichungsmodell $\left(N=187, \chi^{2}=245,26, d f=200, \quad \mathrm{CFI}=0,97\right.$, RMSEA $=0,04, p<0,05)$ ergab, dass die wahrgenommene Motivierungsqualität im Unterricht unter gleichzeitiger Berücksichtigung der Mathematiknote sowohl ein signifikanter Prädiktor für die intrinsische Motivation $(\beta=0,69 ; p<0,01)$ als auch für das Fähigkeitsselbstkonzept der Lernenden $(\beta=0,35 ; p<0,01)$ in Mathematik war (siehe Abb. 1). Schülerinnen und Schüler, die die Motivierungsqualität des Mathematikunterrichts im Vergleich zu ihren Mitschülerinnen und Mitschülern als höher ausgeprägt einschätzten, berichteten im Durschnitt von einer höheren intrinsischen Motivation und einem günstigeren Fähigkeitsselbstkonzept in Mathematik. Die Mathematiknote erwies sich ebenfalls als ein bedeutsamer Prädiktor für beide motivationalen Merkmale, sodass Lernende mit einer durchschnittlich besseren Mathematiknote eine höhere intrinsische Motivation $(\beta=-0,24 ; p<0,05)$ und ein günstigeres Fähigkeitsselbstkonzept $(\beta=-0,58 ; p<0,01)$ im Fach Mathematik auf-

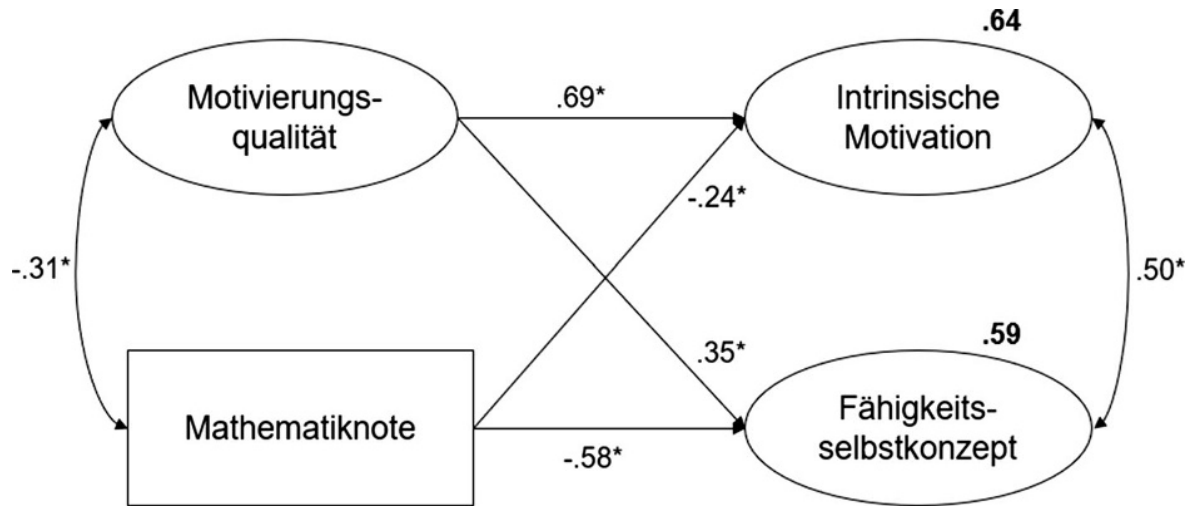

Abb. 1 Strukturgleichungsmodell zur Vorhersage motivationaler Merkmale. Angabe standardisierter $\beta$-Koeffizienten. $\left(\mathrm{R}^{2}=\right.$ fett gedruckte Werte, $* p<0,05$, Notenstufen: $1=$ sehr gut bis $6=$ ungenügend, $N=187$, Modellfit: $\left.\chi^{2}=245,26, d f=200, \mathrm{CFI}=0,97, \mathrm{RMSEA}=0,04\right)$ 
wiesen. Der Effekt der Mathematiknote auf die intrinsische Motivation lässt sich als klein einschätzen, während ein großer Effekt auf das Fähigkeitsselbstkonzept zu beobachten war (Cohen 1992). In dem Modell wurden 64,1\% der Varianz der intrinsischen Motivation und 58,6\% des Fähigkeitsselbstkonzeptes aufgeklärt. Die Hypothesen 1a und 1b wurden anhand der Daten gestützt, sodass die individuell wahrgenommene Motivierungsqualität unter gleichzeitiger Berücksichtigung der Mathematiknote für beide motivationalen Merkmale als bedeutsamer Prädiktor identifiziert werden konnte. ${ }^{3}$

\subsection{Mediation durch das Kompetenzerleben im Unterricht}

Das Strukturgleichungsmodell zur zweiten Forschungsfrage $\left(N=187, \chi^{2}=376,20\right.$, $d f=286, \mathrm{CFI}=0,95, \mathrm{RMSEA}=0,04, p<0,05)$ zeigte, dass das Kompetenzerleben der Lernenden im Unterricht lediglich die Zusammenhänge zwischen der wahrgenommenen Motivierungsqualität bzw. der Mathematiknote und dem Fähigkeitsselbstkonzept mediierte, nicht aber die Effekte für die intrinsische Motivation (siehe Abb. 2). Während sich die individuell erlebte Motivierungsqualität unverändert als bedeutsamer Prädiktor für die intrinsische Motivation der Grundschulkinder erwies $(\beta=0,78, p<0,01)$, wurde der Zusammenhang zwischen der Motivierungsqualität und dem Fähigkeitsselbstkonzept vollständig durch das Kompetenzerleben der Lernenden im Mathematikunterricht vermittelt (indirekter Pfad $\beta=0,41, p<0,05$ ). Lernende, die die Motivierungsqualität des Mathematikunterrichts höher als ihre Mitschülerinnen und Mitschüler einschätzten, fühlten sich im Unterricht kompetenter und schätzten ihre mathematischen Fähigkeiten vergleichsweise höher ein. Ein ähnliches Befundmuster zeigte sich für die Zusammenhänge zwischen der Mathematiknote und dem Fähigkeitsselbstkonzept. Der Effekt der Mathematiknote auf das Fähigkeitsselbstkonzept wurde vollständig über das Kompetenzerleben im Un-

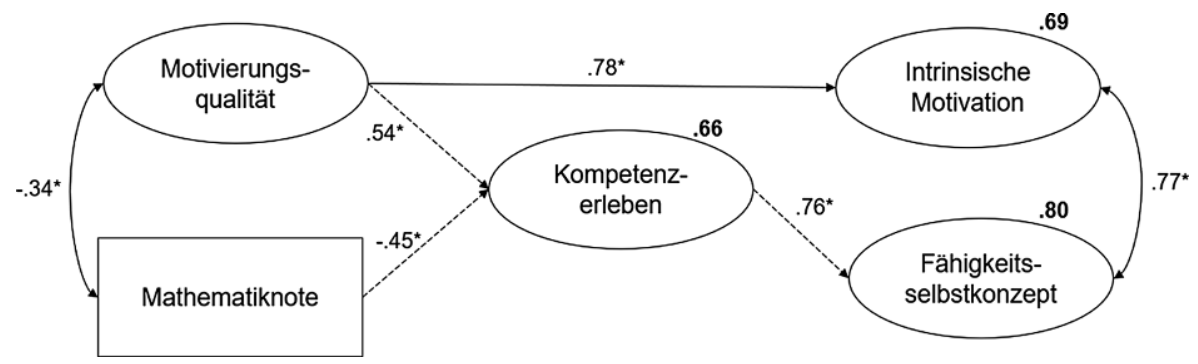

Abb. 2 Strukturgleichungsmodell zur Vorhersage motivationaler Merkmale und Mediation durch Kompetenzerleben. Ausschließlich signifikante Pfade abgebildet, indirekte Pfade in gestrichelter Linie. Angabe standardisierter $\beta$-Koeffizienten. $\left(\mathrm{R}^{2}=\right.$ fett gedruckte Werte, $* p<0,05$, Notenstufen: $1=$ sehr gut bis $6=$ ungenügend, $N=187$, Modellfit: $\chi^{2}=376,20, d f=286, \mathrm{CFI}=0,95, \mathrm{RMSEA}=0,04$ )

\footnotetext{
3 Zusätzliche Analysen zum Vergleich der Bedeutsamkeit beider Prädiktoren zur Vorhersage beider motivationaler Merkmale zeigten, dass sich beide Prädiktoren als ähnlich bedeutsam erwiesen. Dazu wurden Modelle mit gleichgesetzten Pfadgewichten mit freigeschätzten Pfaden vergleichen. Die Restriktionen führten nicht zu signifikant schlechteren Modelfit-Werten (Intrinsische Motivation: $\Delta \chi^{2}=25,05, \Delta d f=1$, $p \geq 0,05$; Fähigkeitsselbstkonzept: $\Delta \chi^{2}=30,09, \Delta d f=1, p \geq 0,05$ ).
} 
terricht mediiert, sodass der indirekte Pfad statistisch signifikant war (indirekter Pfad $\beta=-0,34, p<0,01)$. Der direkte Pfad von der Mathematiknote auf das Fähigkeitsselbstkonzept war nach Aufnahme des Mediators nicht mehr signifikant $(\beta=-0,23$, $p \geq 0,05)$. Diesbezüglich berichteten Grundschulkinder mit durchschnittlich besserer Mathematiknote von einem höheren Kompetenzerleben im Unterricht, das wiederum günstigere Fähigkeitsselbsteinschätzungen im Fach Mathematik prädizierte. Der Zusammenhang zwischen der Mathematiknote und der intrinsischen Motivation war nach Berücksichtigung des Kompetenzerlebens im Unterricht nicht mehr signifikant. Insgesamt lassen sich die indirekten Effekte als klein bis mittel einstufen (Cohen 1992). Der Effekt der wahrgenommenen Motivierungsqualität auf die intrinsische Motivation der Lernenden war groß. In dem Modell wurden 69,2\% der Varianz der intrinsischen Motivation und 79,6\% des Fähigkeitsselbstkonzeptes aufgeklärt. Zudem konnten $65,5 \%$ der Varianz des Kompetenzerlebens der Lernenden erklärt werden. Die Hypothese 2a, in der angenommen wurde, dass dem Kompetenzerleben eine mediierende Funktion für die Zusammenhänge zwischen Motivierungsqualität bzw. Mathematiknote und der intrinsischen Motivation von Lernenden zukommt, konnte empirisch nicht gestützt werden. Hingegen wird die Forschungshypothese $2 \mathrm{~b}$ beibehalten, da sich erwartungskonform zeigte, dass das Kompetenzerleben der Schülerinnen und Schüler im Mathematikunterricht die Zusammenhänge zwischen der individuell wahrgenommenen Motivierungsqualität bzw. der Mathematiknote und dem Fähigkeitsselbstkonzept bedeutsam vermittelte.

\section{Diskussion}

Eine qualitätsvolle Gestaltung des Unterrichts gilt als wichtige Rahmenbedingung für das Lernen von Schülerinnen und Schülern, da sie einen bedeutsamen Beitrag dazu leistet, dass Lernende in Bezug auf das Lernen in akademischen Kontexten eine stärker intrinsisch ausgerichtete Motivation sowie günstigere Fähigkeitsselbstkonzepte entwickeln (Fauth et al. 2014; Praetorius et al. 2018; Rakoczy et al. 2008). Während die positive Verknüpfung der Basisdimensionen von Unterrichtsqualität mit motivationalen Merkmalen bereits vielfach Gegenstand empirischer Forschung war, wurde die Motivierungsqualität des Unterrichts und deren Relevanz für motivationale Merkmale von Schülerinnen und Schülern bislang eher selten untersucht. Die vorliegende Studie hatte zum Ziel, die von Grundschulkindern wahrgenommene Motivierungsqualität des Mathematikunterrichts als spezifische Facette der konstruktiven Unterstützung zu erforschen. Dabei wurden verschiedene motivationale Merkmale als Zielkriterien von Unterricht betrachtet. Die Mathematiknote wurde als weiterer Prädiktor für motivationale Merkmale berücksichtigt. Darüber hinaus wurde das Kompetenzerleben der Lernenden im Unterricht als Mediator der angenommenen Zusammenhänge in den Blick genommen.

Die Ergebnisse zeigten, dass die individuell wahrgenommene Motivierungsqualität im Mathematikunterricht bei gleichzeitiger Berücksichtigung der Mathematiknote als bedeutsamer Prädiktor für die intrinsische Motivation und das Fähigkeitsselbstkonzept identifiziert werden konnte. Im Einklang mit bereits existierenden Befunden für Schülerinnen und Schüler der Sekundarstufe wurde ersichtlich, dass 
auch in der Grundschule eine höher wahrgenommene Motivierungsqualität der Lernenden im Mathematikunterricht mit einer höheren intrinsischen Motivation für das Unterrichtsfach einherging (z. B. Stang et al. im Druck; Thomas und Andreitz 2021). Der Zusammenhang ist dabei anhand theoretisch fundierter Annahmen des heuristischen Angebot-Nutzungsmodells von Klieme und Rakoczy (2008) erklärbar, in dem insbesondere der konstruktiven Unterstützung eine hohe Relevanz für motivationale Merkmale von Lernenden zukommt. Die Befunde der vorliegenden Studie stimmen zudem mit bereits existierenden Studienergebnissen zur Bedeutung der Mathematiknote als einem wichtigen Prädiktor von motivationalen Merkmalen überein (z. B. Möller et al. 2014; Viljaranta et al. 2014). Der Zusammenhang zwischen rückgemeldeten Schulnoten und der intrinsischen Motivation ist dabei damit erklärbar, dass insbesondere gute Noten als positiv erlebte Leistungsrückmeldung Informationen über den Kompetenzstand enthalten und motivierend auf Schülerinnen und Schüler wirken können (Birkel und Tarnai 2018; Ryan und Deci 2020). Dahingegen kann das Internal/External Frame of Reference Model (I/E-Modell) herangezogen werden, um die Relation zwischen Mathematiknote und Fähigkeitsselbstkonzept zu erklären (Marsh 1990).

Das Kompetenzerleben als eine Komponente des Selbstbestimmungserlebens fungierte als bedeutsamer Mediator für die Zusammenhänge zwischen der wahrgenommenen Motivierungsqualität und dem Fähigkeitsselbstkonzept sowie zwischen der Mathematiknote und dem Fähigkeitsselbstkonzept (Ryan und Deci 2020). Dieses Ergebnis ergänzt bestehende Befunde zur Relevanz der Mathematiknote für das Kompetenzerleben als auch für das Fähigkeitsselbstkonzept von Lernenden am Ende der Grundschulzeit (Niepel et al. 2014; Weidinger et al. 2017). Allerdings verschwand der zuvor signifikante Zusammenhang zwischen der Mathematiknote und der intrinsischen Motivation der Lernenden. Dies könnte unter anderem daran liegen, dass der Zusammenhang zwischen der Mathematiknote und der intrinsischen Motivation in dem Analysemodell eher schwach war. Entgegen den Erwartungen wurde der Effekt der Motivierungsqualität auf die intrinsische Motivation der Lernenden im Fach Mathematik nicht über das Kompetenzerleben vermittelt. Dieses Ergebnis lässt sich anhand der Annahmen der Selbstbestimmungstheorie als eher erwartungswidrig einstufen, da ein höheres Kompetenzerleben im Unterricht die intrinsische Motivation der Lernenden im Fach Mathematik begünstigen sollte (Ryan und Deci 2020). Eine mögliche Erklärung für die als nicht bedeutsam zu erkennenden Mediationsprozesse über das Kompetenzerleben könnte darin liegen, dass andere Komponenten des Selbstbestimmungserlebens, wie das Autonomieerleben im Unterricht, eine größere Relevanz für die intrinsische Motivation der Lernenden im Fach Mathematik besitzen könnten, die im Rahmen der Studie jedoch nicht erfasst wurden.

\subsection{Limitationen und Stärken}

Die vorliegende Studie weist einige Limitationen auf. Zum einen basieren die vorgestellten Analysen auf einer querschnittlichen Datengrundlage, sodass keine Aussagen zu der kausalen Wirkrichtung der untersuchten Konstrukte getroffen werden können. Daher sind umgekehrte oder reziproke Wirkrichtungen zwischen der wahrgenommenen Motivierungsqualität und motivationalen Merkmalen ebenfalls denkbar. Um die- 
se Annahme empirisch zu prüfen, wären Längsschnittstudien erstrebenswert. Jedoch lässt sich die Richtung der Zusammenhänge anhand theoretischer Rahmenmodelle begründen, in denen die Wahrnehmung der Lernumwelt aus Sicht der Lernenden als bedeutsame Voraussetzung für die Ausprägung von motivationalen Merkmalen erachtet wird (Eccles und Wigfield 2002; Klieme und Rakoczy 2008; Ryan und Deci 2020). Eine weitere Limitation der vorliegenden Studie liegt in der eher kleinen Stichprobe. Außerdem ist die Qualität der Skala zur Erfassung der individuell wahrgenommenen Motivierungsqualität in Bezug auf die erreichte Reliabilität optimierbar. Möglicherweise spiegelt sich hier zusätzlich eine große interindividuelle Wahrnehmungsvarianz verschiedener Aspekte bei jüngeren Lernenden wider, auf welche bereits die geringe ICC hindeutete. Wahrnehmungsunterschiede könnten beispielsweise auf bestehende Disparitäten der Individuen in Bezug auf die emotionale Bindung zur Lehrkraft oder auf kognitive und affektiv-motivationale Voraussetzungen zurückzuführen sein (Igler et al. 2019; Looser 2011; Ruelmann et al. 2021).

Neben den genannten Limitationen lassen sich ebenfalls wichtige Stärken der Studie identifizieren. Zum einen präsentiert die Studie neu gewonnene Erkenntnisse zur wahrgenommenen Motivierungsqualität des Unterrichts, welche insbesondere dem Forschungsdesiderat des Mangels an empirischen Befunden für Grundschulkinder begegnen. Die Ergebnisse ermöglichen neue Einblicke in die individuell wahrgenommene Motivierungsqualität des Unterrichts aus der Sicht von Lernenden und erweitern somit den bisherigen Kenntnisstand. Dabei wurden die zentralen motivationalen Merkmale, intrinsische Motivation in Mathematik und mathematisches Fähigkeitsselbstkonzept, gemeinsam betrachtet. Eine weitere Stärke der Studie liegt in der Erfassung der Motivierungsqualität mittels Aussagen von Schülerinnen und Schülern. Die Auskunft von Schülerinnen und Schülern zur individuell wahrgenommenen Motivierungsqualität als ein spezifisches Merkmal der konstruktiven Unterstützung im Unterricht gilt als besonders aufschlussreich, da die Wahrnehmung der Unterstützung im Unterricht nicht nur begrenzt durch externe Beurteilende begutachtet werden kann, sondern auch für die Nutzung des Unterrichtangebotes zentral ist (z. B. Clausen 2002; Fauth et al. 2014; Göllner et al. 2018; Vieluf et al. 2020). Allerdings wäre auch eine multiperspektivistische Erforschung der Motivierungsqualität des Unterrichts von besonderem Interesse, um mögliche Unterschiede zwischen den Perspektiven aufzudecken.

\subsection{Implikationen und Schlussfolgerungen}

Aus der vorliegenden Studie lassen sich erste mögliche Implikationen für zukünftige Forschungsarbeiten und die Unterrichtspraxis ableiten. In weiteren Arbeiten könnte die Skala Motivierungsqualität noch facettenreicher erfasst werden, indem beispielsweise zusätzliche Items zur Passung zwischen Anforderungsniveau von Aufgaben und den Fähigkeiten der Lernenden oder zu den Mitbestimmungsmöglichkeiten im Unterricht als Facetten der Motivierungsqualität einbezogen werden. Zudem sollten die Itemformulierungen dahingehend überprüft werden, ob bestimmte Wörter eher schwierig für Grundschulkinder zu verstehen sind und die Einschätzung der Lernenden durch eine „Ich“-Formulierung der Items erleichtert werden könnte. So wäre eine parallele Formulierung zu den Zielkriterien gewährleistet. Eine weitere 
relevante Frage betrifft die differenzielle Wahrnehmung der Motivierungsqualität von Lernenden (z. B. Igler et al. 2019; Ruelmann et al. 2021). Es wäre vorstellbar, dass auch die Relevanz der Motivierungsqualität für motivationale Merkmale differenziell bedeutsam für Lernende unterschiedlicher Ausgangslagen ist. Insbesondere leistungsschwächere Lernende könnten von einer als höher ausgeprägt wahrgenommenen Motivierungsqualität im Unterricht profitieren. Zudem sollte in zukünftigen Studien überprüft werden, ob die vorliegenden Befunde zur Motivierungsqualität des Unterrichts für weitere Unterrichtsfächer, wie dem Deutsch- oder dem Sachunterricht, übertragbar sind. Thomas und Andreitz (2021) zeigten für den weiterführenden Schulkontext, dass bedeutsame Zusammenhänge zwischen dem erlebten motivierenden Umgang im Unterricht und motivationalen Merkmalen für das Fach Deutsch vorlagen. Weitere Studien untermauern diese Befunde für Lernende der 7. Klasse im Fach Deutsch (Stang und McElvany 2020). Allerdings ist unklar, ob diese Zusammenhangsmuster auch im Grundschulkontext vorzufinden sind. Dabei könnten die Erwartungen der Lehrperson, die Relevanz von Noten und die Lernerfahrungen zwischen verschiedenen Unterrichtsfächern bedeutsam variieren und zu abweichenden Befundmustern führen (Dietrich et al. 2015; Eccles und Wigfield 2002). Zukünftig bedarf es Längsschnittstudien, die zusätzlich Aufschluss über die Wirkrichtungen von wahrgenommener Motivierungsqualität und motivationalen Merkmalen von Lernenden geben könnten. Außerdem wären auch Analysen zu anderen Bedürfniskomponenten des Selbstbestimmungserlebens im Unterricht, wie dem Autonomieerleben, als mögliche Mediatoren der Zusammenhänge zwischen der Motivierungsqualität und motivationalen Merkmalen bereichernd. Von Interesse wäre auch, inwiefern die allgemeine Schulfreude einen Einfluss auf die Zusammenhänge zwischen der Motivierungsqualität des Unterrichts und der intrinsischen Motivation der Lernenden hat.

Für die schulische Praxis eröffnen sich ebenfalls erste Anknüpfungspunkte. Insbesondere die bedeutsame Rolle der Motivierungsqualität im Unterricht für das Fähigkeitsselbstkonzept ist ein neuer Befund, der auch für die pädagogische Praxis von hoher Relevanz ist. Da die Unterrichtsgestaltung im Gegensatz zur Notengebung pädagogisch freier gestaltet werden kann, nehmen motivationsbegünstigende Unterrichtsbedingungen einen wichtigen Stellenwert in der Unterrichtspraxis von Lehrkräften ein. Motivationsförderliche Lehr-Lernbedingungen sind außerdem nicht nur kurzfristig für erfolgreiche Lernprozesse, sondern auch auf längere Sicht gesehen für die Entwicklung von Freude und Interesse am Lernen sowie für günstige Fähigkeitsselbsteinschätzungen relevant (Brandenberger et al. 2018; Lazarides et al. 2019). Optimalerweise kann eine hoch ausgeprägte Motivierungsqualität des Unterrichts, ähnlich wie die wahrgenommene konstruktive Unterstützung im Unterricht, zu einer Entschleunigung der eher ungünstigen Entwicklung motivationaler Merkmale beitragen (z. B. Lazarides und Raufelder 2017). Im Unterricht sollte dabei insbesondere auf das Selbstbestimmungserleben der Schülerinnen und Schülern fokussiert werden, um Lernende motivational zu unterstützen (Nie und Lau 2009; Ryan und Deci 2020).

Resümierend bleibt als zentrales Ergebnis der Studie festzuhalten, dass die individuell wahrgenommene Motivierungsqualität im Unterricht bedeutsam mit wichtigen motivationalen Merkmalen von Schülerinnen und Schülern zusammenhing. Ler- 
nende, die die Motivierungsqualität des Mathematikunterrichts im Mittel als höher ausgeprägt einstuften, berichteten zugleich von einer höheren intrinsischen Motivation und einem günstigeren Fähigkeitsselbstkonzept in Mathematik. Der vorliegende Beitrag zur wahrgenommenen Motivierungsqualität aus der Sicht von Lernenden am Ende der Grundschulzeit verdeutlicht, dass die erlebte Motivierungsqualität auch bei gleichzeitiger Berücksichtigung der Mathematiknote mit den beiden untersuchten motivationalen Merkmalen bedeutsam verknüpft war. Die Beschäftigung mit der Motivierungsqualität des Unterrichts stellt ein erstrebenswertes Vorhaben sowohl im forschungsorientierten als auch im schulpraktischen Kontext dar. Auch im Rahmen der universitären Lehramtsausbildung scheint eine Sensibilisierung der angehenden Lehrkräfte für die motivationsrelevanten Bedürfnisse von Schülerinnen und Schülern gewinnbringend zu sein.

Funding Open Access funding enabled and organized by Projekt DEAL.

Open Access Dieser Artikel wird unter der Creative Commons Namensnennung 4.0 International Lizenz veröffentlicht, welche die Nutzung, Vervielfältigung, Bearbeitung, Verbreitung und Wiedergabe in jeglichem Medium und Format erlaubt, sofern Sie den/die ursprünglichen Autor(en) und die Quelle ordnungsgemäß nennen, einen Link zur Creative Commons Lizenz beifügen und angeben, ob Änderungen vorgenommen wurden.

Die in diesem Artikel enthaltenen Bilder und sonstiges Drittmaterial unterliegen ebenfalls der genannten Creative Commons Lizenz, sofern sich aus der Abbildungslegende nichts anderes ergibt. Sofern das betreffende Material nicht unter der genannten Creative Commons Lizenz steht und die betreffende Handlung nicht nach gesetzlichen Vorschriften erlaubt ist, ist für die oben aufgeführten Weiterverwendungen des Materials die Einwilligung des jeweiligen Rechteinhabers einzuholen.

Weitere Details zur Lizenz entnehmen Sie bitte der Lizenzinformation auf http://creativecommons.org/ licenses/by/4.0/deed.de.

\section{Literatur}

Aelterman, N., Vansteenkiste, M., Haerens, L., Soenens, B., Fontaine, J. R. J., \& Reeve, J. (2019). Toward an integrative and fine-grained insight in motivating and demotivating teaching styles: The merits of a circumplex approach. Journal of Educational Psychology, 111(3), 497-521. https://doi.org/10. 1037/edu0000293.

Arens, A. K. (2019). Wertfacetten im Grundschulalter in drei Fächern: Differenzierung, Entwicklung, Geschlechtseffekte und Zusammenhänge zu Noten. Zeitschrift für Pädagogische Psychologie, 35(1), 32-52. https://doi.org/10.1024/1010-0652/a000257.

Arens, A. K., Marsh, H.W., Pekrun, R., Lichtenfeld, S., Murayama, K., \& vom Hofe, R. (2017). Math self-concept, grades, and achievement test scores. Long-term reciprocal effects across five waves and three achievement tracks. Journal of Educational Psychology, 109(5), 621-634. https://doi.org/10. 1037/edu0000163.

Assor, A., Kaplan, H., \& Roth, G. (2002). Choice is good, but relevance is excellent: autonomy-enhancing and surpressing teacher behaviours predicting students' engagement in schoolwork. British Journal of Educational Psychology, 72, 261-278.

Baumert, J., Kunter, M., Blum, W., Brunner, M., Voss, T., Jordan, A., et al. (2010). Teachers' mathematical knowledge, cognitive activation in the classroom, and student progress. American Educational Research Journal, 47(1), 133-180. https://doi.org/10.3102/0002831209345157.

Benning, K., Praetorius, A. K., Janke, S., Dickhäuser, O., \& Dresel, M. (2019). Das Lernen als Ziel: Zur unterrichtlichen Umsetzung einer Lernzielstruktur. Unterrichtswissenschaft, 47(4), 523-545. 
Birkel, P., \& Tarnai, C. (2018). Zensuren und verbale Schulleistungsbeurteilung. In D. H. Rost, J. R. Sparfeldt, \& S. R. Buch (Hrsg.), Handwörterbuch Pädagogische Psychologie (S. 904-916). Weinheim: Beltz.

Brandenberger, C. C., Hagenauer, G., \& Hascher, T. (2018). Promoting students' self-determined motivation in maths: results of a 1-year classroom intervention. European Journal of Psychology of Education, 33(2), 295-317.

Chen, B., Vansteenkiste, M., Beyers, W., Boone, I., Deci, E. L., Van der Kaap-Deeder, J., et al. (2015). Basic psychological need satisfaction, need frustration and need strength across four cultures. Motivation and Emotion, 39(2), 216-236.

Clausen, M. (2002). Unterrichtsqualität. Eine Frage der Perspektive? Empirische Analysen zur Übereinstimmung, Konstrukt- und Kriteriumsvalidität. Berlin, Freie Universität, Dissertation. Münster: Waxmann.

Cohen, J. (1992). A power primer. Psychological Bulletin, 112(1), 155-159. https://doi.org/10.1037/00332909.112.1.155.

Corpus, J. H., McClintic-Gilbert, M. S., \& Hayenga, A. O. (2009). Within-year changes in children's intrinsic and extrinsic motivational orientations: Contextual predictors and academic outcomes. Contemporary Educational Psychology, 34(2), 154-166. https://doi.org/10.1016/j.cedpsych.2009.01.001.

Decristan, J., Hess, M., Holzberger, D., \& Praetorius, A.-K. (2020). Oberflächen- und Tiefenmerkmale. Eine Reflexion zweier prominenter Begriffe der Unterrichtsforschung. In A.-K. Praetorius, J. Grünkorn, \& E. Klieme (Hrsg.), Empirische Forschung zu Unterrichtsqualität. Theoretische Grundfragen und quantitative Modellierungen. Zeitschrift für Pädagogik, (Bd. 66, S. 102-116). Weinheim: Beltz.

Decristan, J., Kunter, M., Fauth, B., Büttner, G., Hardy, I., \& Hertel, S. (2016). What role does instructional quality play for elementary school children's science competence? A focus on students at risk. Journal for Educational Research Online, 8(1), 66-89.

Dietrich, J., Dicke, A.-L., Kracke, B., \& Noack, P. (2015). Teacher support and its influence on students' intrinsic value and effort. Dimensional comparison effects across subjects. Learning and Instruction, 39, 45-54. https://doi.org/10.1016/j.learninstruc.2015.05.007.

Eccles, J. S., \& Wigfield, A. (2002). Motivational beliefs, values, and goals. Annual Review of Psychology, 53(1), 109-132. https://doi.org/10.1146/annurev.psych.53.100901.135153.

Einsiedler, W. (2017). Von Erziehungs- und Unterrichtsstilen zur Unterrichtsqualität. In M. K. W. Schweer (Hrsg.), Lehrer-Schüler-Interaktion: Inhaltsfelder, Forschungsperspektiven und methodische Zugänge (S. 267-287). Wiesbaden: Springer.

Fauth, B., Decristan, J., Rieser, S., Klieme, E., \& Büttner, G. (2014). Student ratings of teaching quality in primary school: dimensions and prediction of student outcomes. Learning and Instruction, 29, 1-9.

Garon-Carrier, G., Boivin, M., Guay, F., Kovas, Y., Dionne, G., Lemelin, J. P., et al. (2016). Intrinsic motivation and achievement in mathematics in elementary school: A longitudinal investigation of their association. Child development, 87(1), 165-175.

Gaspard, H., Dicke, A.-L., Flunger, B., Brisson, B. M., Häfner, I., Nagengast, B., \& Trautwein, U. (2015). Fostering adolescents' value beliefs for mathematics with a relevance intervention in the classroom. Developmental Psychology, 51(9), 1226-1240. https://doi.org/10.1037/dev0000028.

Göllner, R., Wagner, W., Eccles, J.S., \& Trautwein, U. (2018). Students' idiosyncratic perceptions of teaching quality in mathematics: A result of rater tendency alone or an expression of dyadic effects between students and teachers? Journal of Educational Psychology, 110(5), 709-725. https://doi.org/ 10.1037/edu0000236.

Helmke, A. (2012). Unterrichtsqualität und Lehrerprofessionalität: Diagnose, Evaluation und Verbesserung des Unterrichts. Seelze: Klett.

Hochweber, J., Hosenfeld, I., \& Klieme, E. (2014). Classroom composition, classroom management, and the relationship between student attributes and grades. Journal of Educational Psychology, 106(1), 289-300.

Høgheim, S., \& Reber, R. (2015). Supporting interest of middle school students in mathematics through context personalization and example choice. Contemporary Educational Psychology, 42, 17-25.

Hu, L., \& Bentler, P. M. (1999). Cutoff criteria for fit indexes in covariance structure analysis: conventional criteria versus new alternatives. Structural Equation Modeling: A Multidisciplinary Journal, 6(1), $1-55$.

Igler, J., Ohle-Peters, A., \& McElvany, N. (2019). Mit den Augen eines Grundschulkindes: Individuelle Prädiktoren für divergierende Schülereinschätzungen von Unterrichtsqualität. Zeitschrift für Pädagogische Psychologie, 33(3-4), 191-205. 
Jang, H., Kim, E. J., \& Reeve, J. (2012). Longitudinal test of self-determination theory's motivation mediation model in a naturally occurring classroom context. Journal of Educational Psychology, 104, 1175-1188. https://doi.org/10.1037/a0028089.

Jang, H., Reeve, J., \& Deci, E.L. (2010). Engaging students in learning activities: It is not autonomy support or structure but autonomy support and structure. Journal of Educational Psychology, 102, 588-600. https://doi.org/10.1037/a0019682.

Keller, M.M., Goetz, T., Becker, E. S., Morger, V., \& Hensley, L. (2014). Feeling and showing: a new conceptualization of dispositional teacher enthusiasm and its relation to students' interest. Learning and Instruction, 33, 29-38. https://doi.org/10.1016/j.learninstruc.2014.03.001.

Kleickmann, T., Steffensky, M., \& Praetorius, A.-K. (2020). Quality of teaching in science education: more than three basic dimensions? Zeitschrift für Pädagogik, 66(Beiheft 1/20), 37-55.

Klieme, E., \& Rakoczy, K. (2008). Empirische Unterrichtsforschung und Fachdidaktik: Outcome-orientierte Messung und Prozessqualität des Unterrichts. Zeitschrift für Pädagogik, 54(2), 222-237.

Klieme, E., Lipowsky, F., Rakoczy, K., \& Ratzka, N. (2006). Qualitätsdimensionen und Wirksamkeit von Mathematikunterricht. In M. Prenzel, \& L. Allolio-Näcke (Hrsg.), Untersuchungen zur Bildungsqualität von Schule (S. 127-146). Münster: Waxmann.

Klieme, E., Pauli, C., \& Reusser, K. (2009). The Pythagoras Study. Investigating effects of teaching and learning in Swiss and German mathematics classrooms. In T. Janik, \& T. Seidel (Hrsg.), The power of video studies in investigating teaching and learning in the classroom (S. 137-160). Münster: Waxmann.

Krapp, A. (2002). Structural and dynamic aspects of interest development: theoretical considerations from an ontogenetic perspective. Learning and Instruction, 12(4), 383-409.

Krapp, A., \& Hascher, T. (2014). Theorien der Lern- und Leistungsmotivation. In L. Ahnert (Hrsg.), Theorien in der Entwicklungspsychologie (S. 252-281). Heidelberg: Springer.

Kuger, S. (2016). Curriculum and learning time in international school achievement studies. In S. Kuger, E. Klieme, N. Jude, \& D. Kaplan (Hrsg.), Assessing contexts of learning (S. 395-422). Berlin: Springer.

Kunter, M. (2005). Multiple Ziele im Mathematikunterricht. Münster: Waxmann.

Kunter, M., \& Trautwein, U. (2013). Psychologie des Unterrichts. Paderborn: Ferdinand Schöningh.

Kunter, M., \& Voss, T. (2011). Das Modell der Unterrichtsqualität in COACTIV: Eine multikriteriale Analyse. In M. Kunter, J. Baumert, W. Blum, U. Klusmann, S. Krauss, \& M. Neubrand (Hrsg.), Professionelle Kompetenz von Lehrkräften (S. 85-113). Münster: Waxmann.

Kunter, M., Klusmann, U., Baumert, J., Richter, D., Voss, T., \& Hachfeld, A. (2013). Professional competence of teachers: effects on instructional quality and student development. Journal of Educational Psychology, 105(3), 805-820.

Lauermann, F., Tsai, Y.-M., \& Eccles, J.S. (2017). Math-related career aspirations and choices within Eccles et al.'s expectancy-value theory of achievement-related behaviors. Developmental Psychology, 53(8), 1540-1559. https://doi.org/10.1037/dev0000367.

Laufs, A.K., \& Kempert, S. (2021). Außerschulische Interessen als Ressource für die individualisierte Vermittlung der Variablenkontrollstrategie in der Grundschule. Unterrichtswissenschaft, 49, 1-26. https://doi.org/10.1007/s42010-020-00092-6

Lazarides, R., \& Buchholz, J. (2019). Student-perceived teaching quality: how is it related to different achievement emotions in mathematics classrooms? Learning and Instruction, 61, 45-59.

Lazarides, R., \& Ittel, A. (2012). Instructional quality and attitudes toward mathematics: do self-concept and interest differ across students' patterns of perceived instructional quality in mathematics classrooms? Child Development Research. https://doi.org/10.1155/2012/813920.

Lazarides, R., \& Raufelder, D. (2017). Instructional characteristics in mathematics classrooms. Relationships to achievement goal orientation and student engagement. Mathematics Education Research Journal, 29(2), 201-217.

Lazarides, R., Gaspard, H., \& Dicke, A.-L. (2019). Dynamics of classroom motivation: teacher enthusiasm and the development of math interest and teacher support. Learning and Instruction, 60, 126-137. https://doi.org/10.1016/j.learninstruc.2018.01.012.

Lipowsky, F., Rakoczy, K., Drollinger-Vetter, B., Klieme, E., Reusser, K., \& Pauli, C. (2009). Quality of geometry instruction and its short-term impact on students? Understanding of Pythagorean Theorem. Learning and Instruction, 19(6), 527-537.

Little, R. (1988). A test of missing completely at random for multivariate data with missing values. Journal of the American Statistical Association, 83(404), 1198-1202. https://doi.org/10.2307/2290157.

Looser, D. (2011). Soziale Beziehungen und Leistungsmotivation. Die Bedeutung von Bezugspersonen für die längerfristige Aufrechterhaltung der Lern- und Leistungsmotivation. Opladen: Budrich Uni Press. 
MacKinnon, D. P., Fairchild, A. J., \& Fritz, M. S. (2007). Mediation analysis. Annual Review of Psychology, 58, 593-614.

Marsh, H.W. (1990). Causal ordering of academic self-concept and academic achievement: a multiwave, longitudinal panel analysis. Journal of Educational Psychology, 82(4), 646-656. https://doi.org/10. 1037/0022-0663.82.4.646.

Marsh, H. W., Pekrun, R., Lichtenfeld, S., Guo, J., Arens, A. K., \& Murayama, K. (2016). Breaking the double-edged sword of effort/trying hard: Developmental equilibrium and longitudinal relations among effort, achievement, and academic self-concept. Developmental Psychology, 52(8), 1273-1290.

McElvany, N., Köller, O., Bos, W., Gebauer, M.M., Ohle-Peters, A., Schwabe, F., Teerling, A., Igler, J., \& Schlitter, T. (2019). Evaluationsvorhaben von Konzepten und Maßnahmen der fachübergreifenden Leseförderung des Primarbereichs im Rahmen der Bund-Länder-Initiative „Bildung durch Sprache und Schrift“ (BiSS) - Schlussbericht.

Möller, J., Zimmermann, F., \& Köller, O. (2014). The reciprocal internal/ external frame of reference model using grades and test scores. British Journal of Educational Psychology, 84(4), 591-611. https://doi. org/10.1111/bjep.12047.

Musu-Gillette, L., Wigfield, A., Harring, J., \& Eccles, J.S. (2015). Trajectories of change in students' self-concepts of ability and values in math and college major choice. Educational Research and Evaluation, 21(4), 343-370. https://doi.org/10.1080/13803611.2015.1057161.

Muthén, L.K., \& Muthén, B.O. (1998-2017). Mplus User's Guide. Eighth Edition. Los Angeles, CA: Muthén \& Muthén.

Muthén, B. O., \& Satorra, A. (1995). Complex sample data in structural equation modeling. Sociological Methodology, 25, 267-316. https://doi.org/10.2307/271070

Nie, Y., \& Lau, S. (2009). Complementary roles of care and behavioral control in classroom management. The self-determination theory perspective. Contemporary Educational Psychology, 34(3), 185-194.

Niemiec, C.P., \& Ryan, R.M. (2009). Autonomy, competence, and relatedness in the classroom applying self-determination theory to educational practice. Theory and Research in Education, 7(2), 133-144. https://doi.org/10.1177/1477878509104318.

Niepel, C., Brunner, M., \& Preckel, F. (2014). The longitudinal interplay of students' academic self-concepts and achievements within and across domains: Replicating and extending the reciprocal internal/ external frame of reference model. Journal of Educational Psychology, 106(4), 1170-1191. https:// doi.org/10.1037/a0036307.

Ohle, A., \& McElvany, N. (2016). Erfassung von Unterrichtsqualität in der Grundschule: Kognitiver Anspruch, Strukturierung und Motivierungsqualität. In N. McElvany, W. Bos, H. G. Holtappels, M. Gebauer, \& F. Schwabe (Hrsg.), Bedingungen und Effekte guten Unterrichts. Dortmunder Symposium der Empirischen Bildungsforschung, (Bd. 1, S. 117-134). Münster: Waxmann.

Patrick, H., Kaplan, A., \& Ryan, A. M. (2011). Positive classroom motivational environments: convergence between mastery goal structure and classroom social climate. Journal of Educational Psychology, 103(2), 367-382.

Patrick, H., Knee, C. R., Canevello, A., \& Lonsbary, C. (2007). The role of need fulfillment in relationship functioning and well-being: a self-determination theory perspective. Journal of Personality and Social Psychology, 92(3), 434-457. https://doi.org/10.1037/0022-3514.92.3.434.

Pianta, R. C., \& Hamre, B. K. (2009). Conceptualization, measurement, and improvement of classroom processes: standardized observation can leverage capacity. Educational Researcher, 38(2), 109-119.

Pintrich, P. R. (2003). A motivational science perspective on the role of student motivation in learning and teaching contexts. Journal of Educational Psychology, 95(4), 667-686.

Praetorius, A.-K., Klieme, E., Herbert, B., \& Pinger, P. (2018). Generic dimensions of teaching quality. The German framework of Three Basic Dimensions. ZDM, 50(3), 407-426. https://doi.org/10.1007/ s11858-018-0918-4.

Praetorius, A. K., Rogh, W., \& Kleickmann, T. (2020). Blinde Flecken des Modells der drei Basisdimensionen von Unterrichtsqualität? Das Modell im Spiegel einer internationalen Synthese von Merkmalen der Unterrichtsqualität. Unterrichtswissenschaft, 48(3), 303-318. https://doi.org/10.1007/s42010020-00072-w.

Rakoczy, K. (2008). Motivationsunterstützung im Mathematikunterricht - Unterricht aus der Perspektive von Lernenden und Beobachtern. Münster: Waxmann.

Rakoczy, K., Buff, A., \& Lipowsky, F. (2005). Dokumentation der Erhebungs- und Auswertungsinstrumente zur schweizerisch-deutschen Videostudie „Unterrichtsqualität, Lernverhalten und mathematisches Verständnis“. Teil 1. Befragungsinstrumente, In: Materialien zur Bildungsforschung (13), Frankfurt am Main: Gesellschaft zur Förderung Pädagogischer Forschung/Deutsches Institut für Internationale Pädagogische Forschung. 
Rakoczy, K., Klieme, E., \& Pauli, C. (2008). Die Bedeutung der wahrgenommenen Unterstützung motivationsrelevanter Bedürfnisse und des Alltagsbezugs im Mathematikunterricht für die selbstbestimmte Motivation. Zeitschrift für Pädagogische Psychologie, 22(1), 25-35.

Rakoczy, K., Harks, B., Klieme, E., Blum, W., \& Hochweber, J. (2013). Written feedback in mathematics: mediated by students' perception, moderated by goal orientation. Learning and Instruction, 27, 63-73.

Reeve, J., \& Jang, H. (2006). What teachers say and do to support students' autonomy during a learning activity. Journal of Educational Psychology, 98(1), 209-218.

Reeve, J., \& Sickenius, B. (1994). Development and validation of a brief measure of the three psychological needs underlying intrinsic motivation: the AFS scales. Educational and Psychological Measurement, 54(2), 506-515.

Roorda, D. L., Koomen, H. M. Y., Spilt, J. L., \& Oort, F. J. (2011). The influence of affective teacher-student relationships on students' school engagement and achievement. Review of Educational Research, 81(4), 493-529.

Ruelmann, M., Torchetti, L., Zulliger, S., et al. (2021). Kognitiv-motivationale Schüler*innenprofile und ihre Bedeutung für die Schüler*innenwahrnehmung der Lernunterstützung durch die Lehrperson. Unterrichtswissenschaft. https://doi.org/10.1007/s42010-021-00100-3.

Ruzek, E. A., Hafen, C. A., Allen, J.P., Gregory, A., Mikami, A. Y., \& Pianta, R. C. (2016). How teacher emotional support motivates students: the mediating roles of perceived peer relatedness, autonomy support, and competence. Learning and instruction, 42, 95-103.

Ryan, R. M., \& Deci, E.L. (2020). Intrinsic and extrinsic motivation from a self-determination theory perspective: definitions, theory, practices, and future directions. Contemporary Educational Psychology. https://doi.org/10.1016/j.cedpsych.2020.101860.

Sakiz, G., Pape, S. J., \& Hoy, A.W. (2012). Does perceived teacher affective support matter for middle school students in mathematics classrooms? Journal of School Psychology, 50(2), 235-255. https:// doi.org/10.1016/j.jsp.2011.10.005.

Scherer, R., Nilsen, T., \& Jansen, M. (2017). Evaluating individual students' perceptions of instructional quality: an investigation of their factor structure, measurement invariance, and relations to educational outcomes. Frontiers in Psychology, 7, 1-16. https://doi.org/10.3389/fpsyg.2016.00110.

Scherrer, V., \& Preckel, F. (2019). Development of motivational variables and self-esteem during the school career: a meta-analysis of longitudinal studies. Review of Educational Research, 89(2), 211-258.

Schiepe-Tiska, A., Heine, J.-H., Lüdtke, O., Seidel, T., \& Prenzel, M. (2016). Mehrdimensionale Bildungsziele im Mathematikunterricht und ihr Zusammenhang mit den Basisdimensionen der Unterrichtsqualität. Unterrichtswissenschaft, 44(3), 211-225.

Schraw, G., Flowerday, T., \& Lehman, S. (2001). Increasing situational interest in the classroom. Educational Psychology Review, 13(3), 211-224.

Schukajlow, S., \& Krug, A. (2014). Do multiple solutions matter? Prompting multiple solutions, interest, competence, and autonomy. Journal for Research in Mathematics Education, 45(4), 497-533.

Seidel, T., \& Shavelson, R. J. (2007). Teaching effectiveness research in the past decade: the role of theory and research design in disentangling meta-analysis results. Review of Educational Research, 77(4), 454-499. https://doi.org/10.3102/0034654307310317.

Selter, C., Walter, D., Walther, G., \& Wendt, H. (2016). Mathematische Kompetenzen im internationalen Vergleich: Testkonzeption und Ergebnisse. In H. Wendt, W. Bos, C. Selter, O. Köller, K. Schwippert, \& D. Kasper (Hrsg.), TIMSS 2015 - Mathematische und naturwissenschaftliche Kompetenzen von Grundschulkindern in Deutschland im internationalen Vergleich (S. 79-136). Münster; New York: Waxmann.

Sewasew, D., \& Schroeders, U. (2019). The developmental interplay of academic self-concept and achievement within and across domains among primary school students. Contemporary Educational Psychology, 58, 204-212.

Sierens, E., Vansteenkiste, M., Goossens, L., Soenens, B., \& Dochy, F. (2009). The synergistic relationship of perceived autonomy support and structure in the prediction of self-regulated learning. British Journal of Educational Psychology, 79(1), 57-68.

Skaalvik, E.M., \& Skaalvik, S. (2013). School goal structure: associations with students' perceptions of their teachers as emotionally supportive, academic self-concept, intrinsic motivation, effort, and help seeking behavior. International Journal of Educational Research, 61, 5-14.

Stang, J., \& McElvany, N. (2020). Unterschiede in der Wahrnehmung der Qualität des Deutschunterrichts zwischen Grundschülerinnen und Grundschülern. Zeitschrift für Pädagogische Psychologie. https:// doi.org/10.1024/1010-0652/a000275. 
Stang, J., Lepper, C., Steffensky, M., \& McElvany, N. (2020). Einblicke in die Gestaltung des Mathematikund naturwissenschaftsbezogenen Sachunterrichts an Grundschulen in Deutschland. In K. Schwippert, D. Kasper, O. Köller, N. McElvany, C. Selter, M. Steffensky, \& H. Wendt (Hrsg.), TIMSS 2019. Mathematische und naturwissenschaftliche Kompetenzen von Grundschulkindern in Deutschland im internationalen Vergleich (S. 187-208). Münster: Waxmann.

Stang, J., Schwabe, F., \& McElvany, N. (in Druck). Bedeutung des wahrgenommenen motivierenden Umgangs für die intrinsische Lese- und Mathematikmotivation in der Sekundarstufe. In R. Lazarides, \& D. Raufelder (Hrsg.), Motivation in unterrichtlichen fachbezogenen Lehr-Lernkontexten - Perspektiven aus Pädagogik, Psychologie und Fachdidaktiken, Edition ZfE. Wiesbaden: Springer.

Taylor, G., Jungert, T., Mageau, G. A., Schattke, K., Dedic, H., Rosenfield, S., et al. (2014). A self-determination theory approach to predicting school achievement over time: the unique role of intrinsic motivation. Contemporary Educational Psychology, 39(4), 342-358.

Thomas, A.E., \& Andreitz, I. (2021). Entwicklung und Validierung eines Kurzfragebogens zur Erfassung eines motivierenden Unterrichtsstils. Zeitschrift für Pädagogische Psychologie. https://doi.org/ 10.1024/1010-0652/a000304.

Vieluf, S., Praetorius, A.-K., Rakoczy, K., Kleinknecht, M., \& Pietsch, M. (2020). Angebots-NutzungsModelle der Wirkweise des Unterrichts. Ein kritischer Vergleich verschiedener Modellvarianten. Zeitschrift für Pädagogik, 66(Beiheft 1/20), 63-80.

Viljaranta, J., Tolvanen, A., Aunola, K., \& Nurmi, J.-E. (2014). The developmental dynamics between interest, self-concept of ability, and academic performance. Scandinavian Journal of Educational Research, 58(6), 734-756. https://doi.org/10.1080/00313831.2014.904419.

Wang, M. T., \& Eccles, J. S. (2013). School context, achievement motivation, and academic engagement: a longitudinal study of school engagement using a multidimensional perspective. Learning and Instruction, 28, 12-23.

Weidinger, A. F., Steinmayr, R., \& Spinath, B. (2017). Math grades and intrinsic motivation in elementary school: a longitudinal investigation of their association. British Journal of Educational Psychology, 87(2), 187-204.

Weidinger, A. F., Steinmayr, R., \& Spinath, B. (2018). Changes in the relation between competence beliefs and achievement in math across elementary school years. Child Development, 89(2), 138-156. https:// doi.org/10.1111/cdev.12806.

Weidinger, A. F., Steinmayr, R., \& Spinath, B. (2019). Ability self-concept formation in elementary school: no dimensional comparison effects across time. Developmental Psychology, 55, 1005-1018. https:// doi.org/10.1037/dev0000695.

Wentzel, K.R., Battle, A., Russell, S.L., \& Looney, L.B. (2010). Social supports from teachers and peers as predictors of academic and social motivation. Contemporary Educational Psychology, 35(3), 193-202. 